\title{
SPERM MALFORMED ULTRASTRUCTURE IN GOSSYPOL-TREATED RABBITS
}

\author{
W.F. Shaaban, T. A. Taha, F. D. El-Nouty and M. H. Salem \\ Department of Animal Production, Faculty of Agriculture (El-Shatby), Alexandria University, \\ Alexandria, Egypt
}

\begin{abstract}
SUMMARY
This study aimed to evaluate the effect of a sublethal dose of gossypol (20 mg/kg BW, every other day) on sperm malformed ultrastructure of male rabbits. The experiment lasted 8 weeks. Gossypol administration exhibited: severe deteriorations in the head, connecting piece and midpiece of the sperm; significant increases in the malformed acrosomes; dramatic destructions expressed as degeneration of cytoplasmic membrane and disappearance of some segmented columns; and significant increases in the incidence of cytoplasmic droplets. Other striking defects were also observed in the midpiece, especially disappearance of the cytoplasmic membrane, extensive vacuolation, loss of cristae and interruption in the continuity of the mitochondria along the length of the midpiece, as well as complete disappearance of some of outer dense fibers and of the nine-fiber doublets. In conclusion, gossypol treatment induced reproductive toxicity which alters the sperm morphogenesis and producing serious and deleterious malformed sperm ultrastructures.
\end{abstract}

Keywords: Gossypol, sperm ultrastructure, acrosome, axonemal fiber, mitochondrial sheath

\section{INTRODUCTION}

Reduction in sperm motility has been reported in several animal species following treatment with gossypol (Randel et al., 1992; Taha et al., 2006). Chenoweth et al. (1994) suggested that impairment of sperm motility by gossypol treatment may be due to the structural damage to the sperm midpiece. Electron microscopy of bull sperm treated with gossypol revealed gaps in the mitochondrial helix of the midpiece (Velasquez-Pereira et al., 1998), and the same was also reported in rats (Oka and Hrudka, 1982). Furthermore, electron microscopy of rat and bull spermatozoa revealed gossypol-induced extensive mitochondrial damage presented as extensive vacuolation and loss of cristae (Swan et al., 1990; and Velasquez-Pereira et al., 1998). Randel et al. (1992) suggested that this defect may be responsible for the observed reduction in sperm motility of animals treated with gossypol. Axonemal deficiencies are often the cause of lowered motility, progressive motility and fertility of the spermatozoa (Williamson et al., 1984; Hancock and de Kretser, 1992), and abnormalities or reduction in the number of axonemal fibers could have significant effects on the progressive motility of the spermatozoon (Hancock and de Kretser, 1992; and Zavos et al., 1998). One of the most striking changes in the axoneme of the gossypol-treated rats was the displacement of most of the outer dense fibers (Hoffer, 1983), as well as the disarrangement of the dense fibers and axial microtubules of the tail (Swan et al., 1990).

Our recent studies clearly indicated that gossypol has pronounced hazardous effects on seminal characteristics (Taha et al., 2006) and seminal plasma constituents (Shaaban et al., 2008; Taha et al., 2008) in male rabbits, which may lead to deleterious productive and reproductive performance. A malformed acrosome of the gossypol-treated rat sperm was also noted indicating its dissolution and detachment from the underlining nucleus (Oka and Hrudka, 1982; and Hoffer, 1983). Moreover daily ingestion of gossypol provokes infertility in various animal species, including humans. The contraceptive effect of gossypol in man was discovered first in China, and still continues to be tested as a favorable candidate as a male contraceptive (Cui et al., 2004). Thus the present study was carried out to evaluate the malformed ultrastructure arrangement of sperm in gossypol-treated rabbits.

\section{MATERIALS AND METHODS}

\section{Animals and management:}

This study was carried out at the Department of Animal Production, Faculty of Agriculture, Alexandria University, Alexandria, Egypt. Experiments were carried out after the Departmental approval, and were done without any commercial profit purposes for the Authors or the Department. All procedures and experimental protocols were conducted in accordance with the "Guide for the Care and Use of Laboratory Animal" prepared by the Institute for Laboratory Animals Resources (ILAR) of the National Academy of Science, National Academy Press, Washington, D.C. (1996). The present experiment was carried out to study the effect 
of gossypol on malformed ultrastructure arrangement of rabbit sperm. Rabbits were chosen as an experimental animal owing to the fact that they are excellent model for reproductive toxicological studies. This is because the male rabbit is the smallest, least expensive animal that can be ejaculated with an artificial vagina, where serial semen samples could be obtained for biochemical and fertility evaluations (Foote and Carney, 2000). Gossypol was extracted from cottonseeds and purified according to Boatner (1948) as described by Taha et al. (2006). Gossypol was firstly dissolved in acetone then in corn oil to give a concentration of $20 \mathrm{mg} / \mathrm{ml}$; this mixture was freshly prepared on the day of dosing.

Ten mature male New Zealand White rabbits (from the Agricultural Experimental Station, Faculty of Agriculture, Alexandria University, Egypt) aged 6-8 months and weighing $2.68 \pm 0.14 \mathrm{~kg}$ at the beginning of the experiment were used during the reproduction season (starting in September to avoid summer heat stress, as rabbit semen characteristics vary among seasons where increasing ambient temperature adversely affects semen quality (Hafez and Hafez, 2000). The rabbits were individually housed in cages. Feed and water were provided ad libitum. The animals were fed pellets consisting of (per $\mathrm{kg}$ ) $330 \mathrm{~g}$ berseem (Trifolium alexandrinum) hay, $170 \mathrm{~g}$ soybean meal, $165 \mathrm{~g}$ grounded corn, $160 \mathrm{~g}$ barley, $120 \mathrm{~g}$ wheat bran, $38 \mathrm{~g}$ molasses, $10 \mathrm{~g}$ salt, $4 \mathrm{~g}$ dicalcium phosphate, and $3 \mathrm{~g}$ vitamins. The chemical analysis of the diet according to Association of Official Analytical Chemists (1995) indicated that it contained (per $\mathrm{kg}$ ) $175 \mathrm{~g}$ crude protein, $140 \mathrm{~g}$ crude fiber and $27 \mathrm{~g}$ fat. All animals were allowed to adjust to their new environment and tested for semen quality through three weeks before the experiment began.

\section{Experimental design:}

The rabbits were randomly divided into two groups of five animals each, and were assigned at random to one of the following treatments: the first group served as control (the animals were given an equivalent dose of the vehicle consisting of corn oil + acetone); the second group was used to study the effect of gossypol $\left(1 / 20 \mathrm{LD}_{50}, 20 \mathrm{mg} / \mathrm{kg} \mathrm{BW}\right)$. The proper dose for each rabbit was given orally with the help of a syringe directly into the esopharyngeal region. All animals were dosaged every other day throughout the treatment period, which extended for eight weeks (i.e. 56 days which is almost equal to the duration of spermatogenesis in the rabbit (52 days) [Swierstra and Foote, 1965]). Semen collection was carried out weekly from all animals throughout the 8 -week experimental period. Ejaculates were obtained using an artificial vagina and a teaser doe and seminal characteristics were evaluated (Taha et al., 2006).

\section{Electron microscopy:}

At the end of the treatment period, spermatozoa obtained from the control and gossypol-treated groups were used to study the effect of gossypol on the ultrastructural features of rabbit sperm.

\section{Transmission electron microscopy:}

All chemicals used in this study were purchased from Sigma (Sigma Chemical Co., St. Louis, MO, USA). Freshly collected semen specimens were washed twice by centrifugation (800 $\mathrm{xg}$ for $10 \mathrm{~min}$ ) and resuspended using phosphate-buffered saline medium. Transmission electron microscopy (TEM) specimens were fixed in glutaraldehyde (3\%) buffered with $0.15 \mathrm{M}$ cacodylate at $\mathrm{pH}$ 7.4. They were post-fixed in osmium tetroxide (1\%), dehydrated in graded alcohol solutions, and embedded in Epon 812. Ultra thin sections were cut with glass knife on a Leica EMKMR2 ultramicrotome, England, stained with uranyl acetate and lead citrate, and examined under a JEOL 100 CX transmission electron microscope (Japan).

\section{Scanning electron microscopy:}

Semen samples were processed and prepared for Scanning Electron Microscope (SEM) examination by the methods of Hafez and Kanagawa (1973). The dried samples were mounted on brass stubs and coated with gold using ion sputtering device JFC-1100E JEOL, Japan. The specimens were examined at $25 \mathrm{kv}$ with JEOL JSM-5300 Scanning Electron Microscope (Japan).

\section{Statistical analysis:}

Data were analyzed using generalized linear model procedure (SAS, 1999) by twoway ANOVA. Comparisons among main effects were tested using LSD $_{.05}$ of SAS (1999).

\section{RESULTS}

Data on the ultrastructural assessment of spermatozoa from control and gossypol-treated male rabbits are indicated in Table 1 . The ultrastructural features of the spermatozoa obtained from these animals at the end of treatment period are presented in Figs. 1 to 5 .

\section{Acrosomal defects:}

Significant increases $(P<0.01)$ in the numbers of reacted acrosomes were noted following gossypol treatment (Table 1). Electron micrographs (Figures 1, A and C) display a normal rabbit sperm showing an intact acrosome; while the malformed 
acrosome of gossypol-treated rabbit sperm (Figures 1, B and D) reveal its dissolution and detachment from the underlying nucleus.

\section{Connecting piece anomalous:}

Gossypol administration exhibited severe deteriorations in the connecting piece (Table 1), which was illustrated by transmission electron micrographs. Normal rabbit sperm revealing intact connecting piece with normal appearance of cytoplasmic membrane and mitochondrial sheath is displayed in Figure 2A, while gossypol-treated rabbit sperm exhibited lesion of the cytoplasmic membrane, disappearance of some segmented columns (Figure 2B) and degeneration in the mitochondrial sheath near the connecting piece (Figure 2C).

\section{Midpiece defects:}

The most striking changes observed in the sperm of gossypol-treated rabbits were defects in the midpiece (Table 1), especially degeneration of the mitochondrial sheath. Transmission electron micrographs of longitudinal sections of normal rabbit sperm through midpiece and principal piece (Figure 3A) indicated normal appearance of cytoplasmic membrane and mitochondrial sheath; while sperm of the gossypol-treated rabbits (Figures 3, B and $\mathrm{C}$ ) revealed disappearance of cytoplasmic membrane and interruption in the continuity of the mitochondria along the length of the midpiece (Figure 3C) but the principal piece seems normal (Figure 3B). On the other hand, the ultrastructural arrangement of cross section in the midpiece of normal rabbit sperm (Figure 4A) displayed that cytoplasmic membrane, mitochondrial sheath, outer dense fibers and axoneme are all normal in their appearance. However, sperm of gossypol- treated rabbits (Figure 4, B and C) revealed rupture of cytoplasmic membrane and extensive vacuolation of mitochondria (Figure 4C). The most severe abnormality noted in the axoneme of sperm from gossypol-treated rabbits was the complete disappearance of some of the ninefiber doublets and outer dense fibers (Figure 4C).

\section{Cytoplasmic droplets:}

Gossypol treatment was also associated with significantly higher $(P<0.01)$ incidence of cytoplasmic droplets (Table 1). Scanning electron micrographs of the gossypol-treated rabbit sperm revealed the presence of cytoplasmic droplet (Figure 5A). Transmission electron micrographs of longitudinal (Figure 5C) and cross-section (Figure 5B) of the cytoplasmic droplet, surrounding the midpiece clearly defined the extensive vacuolation and loss of cristiae along the length of the mitochondrial sheath. The presence of numerous large vesicles in the cytoplasmic droplet and its hollow appearance was also observed (Figures 5, B and C).

\section{DISCUSSION}

To express its fertility potential and fulfill its reproductive function, the spermatozoa must be viable and must possess an efficient motor apparatus to drive them through the relatively large distance separating the vagina from the oviduct, and an intact acrosome to acquire fertilizing competence through capacitation. At the ultrastructural level, the sperm tail provides the spermatozoon with means of motility and is composed of the neck and the middle, principal, and end-pieces. The entire length of the sperm tail or axoneme is composed of nine pairs (doublets) of filaments or microtubules that are arranged radially around two central filaments $(9+2$ arrangement). This arrangement of filaments is surrounded by nine outer dense fibers. The outer dense fibers appear to be associated with the nine pairs of filaments. Furthermore, the entire midpiece is covered by mitochondria that generate the energy needed for sperm motility.

The toxicity of gossypol on rabbit spermatozoa had not been previously evaluated at the ultrastructural level. In the present study, the effect of gossypol on sperm structures and specifically on the axoneme (of the sperm tail) at the level of the middle and principal-pieces was evaluated. The study revealed a malformed acrosome of the gossypol-treated rabbit sperm indicating its dissolution and detachment from the underlying nucleus (Figures 1, B and D), which is in agreement with previous studies in other species (Oka and Hrudka, 1982; Hoffer, 1983).

Several hypotheses had been proposed to explain the detrimental effects of gossypol at the ultrastructural level of spermatozoa: first, structural integrity of numerous sperm structures depends on $\mathrm{S}-\mathrm{S}$ cross-linked polypeptides. Disturbance of this bond may be associated with damage to the midpiece mitochondria (Baccetti et al., 1986); second, damage due to lipid peroxidation in human sperm has been associated with loss of membrane integrity (Aitken and Fisher, 1994) and; third, gossypol promotes the formation of reactive oxygen species (Barhoumi and Burghardt, 1996).

Reduction in sperm motility has been reported in several animal species treated with gossypol (Randel et al., 1992; and Wang et al., 1992). Chenoweth et al. (1994) suggested that motility may be impaired by structural damage to the sperm midpiece in animals treated with 
gossypol. Electron microscopy of bull sperm treated with gossypol revealed gaps in the mitochondrial helix of the midpiece (Velasquez-Pereira et al., 1998), as well as in rats (Oka and Hrudka, 1982). Furthermore, electron microscopy revealed extensive mitochondrial damage which was presented as extensive vacuolation and loss of cristae (Velasquez-Pereira et al., 1998). Also Randel et al. (1992) suggested that this defect may be responsible for the observed reduction in sperm motility in animals treated with gossypol. Collectively, these findings are in agreement with the present results which revealed an interruption in the continuity of the mitochondria along the length of the midpiece (Figures 3, B and C), besides several mitochondria were severely vacuolated or entirely demolished. The present observations are consistent with those of Hoffer (1983) and Swan et al. (1990).

Axonemal deficiencies are often the cause of lowered mass motility, progressive motility and fertility in spermatozoa (Williamson et al., 1984; Hancock and de Kretser, 1992), where abnormalities or reduction in the number of axonemal fibers could have significant effects on the progressive motility of the spermatozoon (Hancock and de Kretser, 1992; Zavos et al., 1998). One of the most striking changes in the axoneme of gossypol-treated rats was the displaced outer dense fibers (Hoffer, 1983), in addition to the disarrangement of the outer dense fibers and axial microtubules of the tail (Rovan et al., 1984; and Swan et al., 1990). These findings are consistent with the present observations in which the most severe abnormality noted in the axoneme of spermatozoa from gossypol-treated specimens was the complete disappearance of one or more of the outer dense fibers and one or more of the nine fiber doublets (Figure 4C).

Outer dense fibers are composed of several prominent proteins which have been assembled along the axoneme by means of "leucine zippers" (Shao et al., 1999). Furthermore, it has been demonstrated that all leucine residues in upstream leucine zippers are required for interaction between outer dense fibers proteins (Shao et al., 1997). In addition, maximal incorporation of Ieucine into the outer dense fibers polypeptides occurs during spermiogenesis (Vera et al., 1987). Moreover, Comparison of sperm tail outer dense fibers proteins of rat, rabbit and humans indicated structural similarity of sperm tail outer dense fibers among these different species (Kim et al., 1999). Leucine zipper, one of these transcription factors, is rich in basic amino acids (Lysine and Arginine) and contains four or five Ieucine residue spaces at intervals of seven amino acids (Cooper, 1997). Our previous studies on the same animals (Taha et al., 2008) revealed that treatment with the same dose of gossypol reduced the levels of lysine, arginine and leucine up to $50 \%$. The severe reductions in the levels of these amino acids which represent the main contributors of the transcription factor (leucine zipper) seem to hinder the synthesis or function of this transcription factor which in turn disturbs the regulation of gene expression. These findings are consistent with previous studies in which gossypol induced inactivation of transcription factors in rat spermatogenic cells (Teng, 1992; and Teng et al., 1995). Due to the importance of the leucine zippers in sperm tail outer dense fiber protein interactions, the reducing effect of gossypol on lysine, arginine and leucine could explain and might be the reason for the observed disappearance of the outer dense fibers. Previous reports indicated that the contents of tubulin and its motor protein (dynein) in spermatogenic cells are simultaneously reduced after gossypol treatment (Teng, 1995; and 1997). Also, gossypol causes inhibition of various enzyme activities involved in tubulin gene expression and tubulin synthesis (Strom-Hansen et al., 1989; and Gonzalez-Garza et al., 1993). In addition, gossypol alters the structure of intracellular membrane which impairs the m-RNA transportation system and reduces the rate of tubulin synthesis (Reyes et al., 1984; and de Peyster et al., 1986). Furthermore, gossypol binds to tubulin at a specific site and consequently prevents the tubulin from the assembling into microtubules (Medrano and Andreu, 1986). The unassembled free tubulin binds to nascent tubulin polypeptides on ribosomes, blocking further elongation and causing degradation of the tubulin m-RNA (Cleveland, 1989; Amos and Amos, 1991). These observations have validated gossypol as a potential inhibitor for the synthesis of contractile apparatus in the spermatogenic cell.

The present ultrastructural studies on gossypol-treated rabbits also reveal missing segments of sperm plasma membrane, which is consistent with several previous reports (Rovan et al., 1984; and Swan et al., 1990). Gossypol can alter the properties of lipid membranes that can modify protein function and structure (Cuéllar and Ramirez, 1993). Barhoumi and Burghardt (1996) reported that gossypol promoted the formation of reactive oxygen species and the depletion of glutathione in rat hepatocytes. Bender et al. (1988) reported a reduction in the antioxidants in the testes of rats fed gossypol. Damage due to lipid peroxidation in human sperm has been associated with loss of membrane integrity (Aitken and Fisher, 1994). 
In mammalian spermatozoa cytoplasmic droplets of various sizes are noted in the neck region as one of the abnormalities of ejaculated spermatozoa. In most mammalian species the incidence of cytoplasmic droplets is relatively small, and during the maturation processes of the spermatozoon in the epididymis, the droplet migrates caudally and ultimately disappears. In the spermatozoa of domestic animals, the presence of a cytoplasmic droplet in the neck region is considered as a sign of immaturity. In the cauda epididymidis, the major absorption of the proteins synthesized in the preceding parts of the epididymidis to the migrating sperm cells takes place, but it is probably also the site of the synthesis of enzymes which destroy unwanted or undesirable components of the spermatozoa. Unwanted components already collect in the cytoplasmic droplets from the testis (where formation of the droplet begins). In bulls, cauda epididymidis is the site of initial synthesis of seminal ribonuclease (Matoušek et al., 1981) which, apparently by adsorption, inactivates undesirable RNA in the cytoplasmic droplets (Matoušek et al., 1980 a). The synthesis of seminal ribonuclease depends on the blood plasma testosterone concentration where reduced testosterone synthesis leads to loss of seminal ribonuclease synthesis in the cauda epididymidis (Matoušek et al., 1980 a; Matoušek et al., 1981). Another substance isolated from bull seminal plasma is phospholipid-binding protein (PBP), which releases the cytoplasmic droplets from epididymal sperm cells (Matoušek and Kysilka, 1980). The synthesis of PBP is dependent on the blood plasma testosterone concentration (Matoušek et al., 1980 b). Functionally, seminal plasma PBP is not species-specific, so it releases the cytoplasmic droplets from ram, boar and rabbit sperm cells. The synthesis of both seminal ribonuclease and seminal phospholipid-binding protein are dependent on the blood plasma testosterone concentration. Thus the suppression of the synthesis of both seminal proteins by gossypol treatment seems to be attributed to the gossypol-induced reduction in testosterone concentration noted in our previous studies on rabbits (Taha et al., 2006). This reduction in the synthesis of both proteins seems to suppress the release of cytoplasmic droplets from epididymal sperm cells (Figures 5, A C).

It has been shown that the cytoplasmic droplet contains lipids, carbohydrates and lysomal and glycolytic enzymes, and is made up of components of the endoplasmic reticulum mixed with remnants of the disintegrating Golgi complex in the residual cytoplasm (Dott and Dingle, 1968). Gomez et al. (1996) reported that the link between semen quality and the reactive oxygen species (ROS) generation lies in the presence of excess residual cytoplasm. Thus when spermatogenesis is impaired, the cytoplasmic extrusion mechanisms are defective and spermatozoa are released from the germinal epithelium carrying surplus residual cytoplasm. Under these circumstances, the spermatozoa released during spermiation are thought to be immature and functionally defective (Huszar et al., 1997). Therefore, when the cytosolic space is increased through the retention of excess residual cytoplasm, the resultant greater activity of the glucose-6phosphate dehydrogenase leads to the excessive generation of NADPH and hence to the over-production of ROS. The latter then initiates a lipid peroxidation cascade and participates in a loss of sperm function (Aitken et al., 1997).

In conclusion, gossypol induced sperm malformed ultrastructure expressed as sperm membrane injuries, acrosome reaction, gaps in the mitochondrial helix of the midpiece, disappearance of some segmented columns and reduction in the number of axonemal fibers. This could be partially attributed to reduction in the level of some amino acids which seems to hinder the synthesis or function of some transcription factors (leucine zipper). This in turn disturbs the regulation of gene expression as reflected in axonemal deficiencies. Furthermore, gossypol directly promotes the formation of reactive oxygen species (ROS) and indirectly impairs spermatogenesis resulting in deficiency in cytoplasmic extrusion mechanisms which leads to overproduction of ROS. Collectively, the excessive generation of ROS initiates a lipid peroxidation cascade.

\section{REFERENCES}

Aitken, J. and H. Fisher, 1994. Reactive oxygen species generation and human spermatozoa: The balance of benefit and risk. Bio. Essays 16: 259-267.

Aitken, R.J., H.M. Fisher, N. Fulton, E. Gomez, W. Knox, B. Lewis and D.S. Irvine, 1997. Reactive oxygen species generation by human spermatozoa is induced by exogenous NADPH and inhibited by the flavoprotein inhibitors diphenylene iodonium and quinacrine. Mol. Reprod. Dev. 47: 468-482.

Amos, L. and W. Amos, 1991. Molecules of the Cytoskeleton. The Guilford Press: New York.

A.O.A.C., 1995. Association of Official Analytical Chemists. Official Methods of Analysis. $16^{\text {th }}$ ed., VA, Arlington. 
Baccetti, B.E., A. Bigliardi, T. Burrini, T. Renieri and G. Selmi, 1986. The action of gossypol on rat germinal cells. Gamete Res. 13: 1-17.

Barhoumi, R. and R.C. Burghardt, 1996. Kinetic analysis of the chronology of patulin- and gossypol-induced cytotoxicity in vitro. Fundam. Appl. Toxicol. 30: 290297.

Bender, H.S., S.Z. Derolf and H.P. Misra, 1988. Effect of gossypol on the antioxidant defense system of the rat testis. Arch. Androl. 21: 59-70.

Boatner, C. H., 1948. Pigments of cottonseed. pp. 215-223. in Cottonseed and Cottonseed Products. Their Chemistry and Chemical Technology. A. E. Bailey, Ed. Wiley, Interscience, New York.

Chenoweth, P.J., C.A. Risco, R.E. Larsen, J. Velez, N. Shaw, C.C. Chase and T. Tran, 1994. Effects of dietary gossypol on aspects of semen quality, sperm morphology and sperm production in young Brahman bulls. Theriogenology 42: $1-13$.

Cleveland, D.W., 1989. Autoregulated control of tubulin synthesis in animal cells. Curr. Opinion Cell Biol. 1: 10-14.

Cooper, G.H., 1997. The Cell: A Molecular Approach. ASM Press: Washington, D.C.

Cuéllar, A. and J. Ramirez, 1993. Further studies on the mechanism of action of gossypol on mitochondrial membrane .Int. J. Biochem. 25: 1149-1155.

Cui, G.H., Z.L. Xu, Z.J. Yang, Y.Y. Xu and S.P. Xue, 2004. A combined regimen of gossypol plus methyltestosterone and ethinylestradiol as a contraceptive induces germ cell apoptosis and expression of its related genes in rats. Contraception 70: 335-342.

de Peyster, A., P. Hysop, C. Kuhn and R. Sauerheber, $1986 . \quad$ Membrane structure/functional perturbations induced by gossypol: effects on membrane order, liposome permeability and insulin-sensitive hexose transport. Biochem. Pharmacol. 35: 3391-3399.

Dott, H.M. and J.T. Dingle, 1968. Distribution of lysosomal enzymes in the spermatozoa and cytoplasmic droplets of bull and ram. Expl. Cell Res. 52: 523-540.

Foote, R.H. and E.W. Carney, 2000. The rabbit as a model for reproductive and developmental toxicity studies. Reprod. Toxicol. 14: 477-493.

Gomez, E., D.W. Buckingham, J. Brindle, F. Lanzafame, D.S. Irvine and R.J. Aitken, 1996. Development of an image analysis system to monitor the retention of residual cytoplasm by human spermatozoa: correlation with biochemical markers of the cytoplasmic space, oxidative stress and sperm function. J. Androl. 17: 276-287.

Gonzalez-Garza, M., S. Matlin, B. MataCardenas and S. Said-Fernadez, 1993. Inhibition of malic enzyme and alcohol dehydrogenase by (+/-), (+), and (-) gossypol. Arch. Med. Res. 24: 183-187.

Hafez, E. S. E. and B. Hafez, 2000. Reproduction in Farm Animals. $7^{\text {th }}$ ed. Lipooincott Williams and Wilkins: Philadelphia.

Hafez, E.S.E. and H. Kanagawa, 1973. Scanning electron microscopy of human, monkey and rabbit spermatozoa. Fert. Steril. 24: 776-787.

Hancock, A.D. and D.M. de Kretser, 1992. The axonemal ultrastructure of spermatozoa from men with asthenospermia. Fertil. Steril. 57: 661-664.

Hoffer, A.P., 1983. Effects of gossypol on the seminiferous epithelium in the rat: A light and electron microscope study. Biol. Reprod. 28: 1007-1020.

Huszar, G., M. Sbracia, L. Vigue, D.J. Miller and B.D. Shur, 1997. Sperm plasma membrane remodeling during spermiogenetic maturation in men: relationship among plasma membrane beta 1,4-galactosyltransferase, cytoplasmic creatine phosphokinase, and creatine phosphokinase isoform ratios. Biol. Reprod. 56: 1020-1024.

Kim, Y.H., J.R. McFarlane, M.K. O’Bryan, G. Almahbobi, P.D. Temple-Smith and D.M. de Kretser, 1999. Isolation and characterization of rat sperm tail outer dense fibers and comparison with rabbit and human spermatozoa using a polyclonal antiserum. J. Reprod. Fertil. 116: 345-353.

Matoušek, J. and Č. Kysilka, 1980. The haemolytic factor (phospholipid-binding protein) of the bull reproductive tract- its synthesis and effect on spermatozoal cytoplasm droplets. Anim. Reprod. Sci. 3: 195206.

Matoušek, J., J. Pícha, D. Píchová and J. Petelíková, 1980 a. Seminal ribonuclease of bulls - biosynthesis In normal and orchitic bull and correlation with blood plasma hormone levels. Anim. Reprod. Sci. 3: 116.

Matoušek, J., J. Pícha and D. Píchová, 1980 b. Synthesis of phospholipid-binding protein in the reproductive organs of the bull during ontogenesis. Anim. Reprod. Sci. 3, 207-216.

Matoušek, J., J. Pícha and D. Píchová, 1981. Seminal ribonuclease synthesis in bull reproductive organs during ontogenesis. Anim. Reprod. Sci. 4: 9-18.

Medrano, F. and J. Andreu, 1986. Binding of gossypol to purified tubulin and inhibition 
of its assembly into microtubules. Eur. J. Biochem. 158: 63-69.

Oka, R. and F. Hrudka, 1982. Segmental aplasia of the mitochondrial sheath and sequelae induced by gossypol in rat spermatozoa. Bio. Reprod. 26: 183-195.

Randel, R.D., C.C. Chase and S.J. Wyse, 1992. Effects of gossypol and cottonseed products on reproduction of mammals. J. Anim. Sci. 70: 1628-1638.

Reyes, J., J. Allen, N. Tauphaichitr, A. Bellve and D. Benos, 1984. Molecular mechanisms of gossypol action on lipid membrane. J. Biol. Chem. 259: 9607-9615.

Rovan, E., N.R. KaIIa, J. Frick and H. Adam, 1984. Effect of gossypol on bull spermatozoa in vitro. Urol. Res. 12: 187192.

SAS, 1999. SAS user's guide. Statistics, version $8^{\text {th }}$ ed. SAS Institute, Cary, NC.

Shaaban, W.F., T.A. Taha, F.D. EL-Nouty, A.R. EL-Mahdy and M.H. Salem, 2008. Reproductive toxicologic effects of gossypol on male rabbits: biochemical, enzymatic, and electrolytic properties of seminal plasma. Fertil. Steril. 89: 15851593.

Shao, X., H.A. Tarnasky, J.P. Lee, R. Oko and F.A. van der Hoorn, 1999. Spag4, a novel sperm protein, binds outer dense-fiber protein Odf1 and localizes to microtubules of manchette and axoneme. Dev. Biol. 211: 109-123.

Shao, X., H.A. Tarnasky, U. Schalles, R. Oko and F.A. van der Hoorn, 1997. Interactional cloning of the $84-\mathrm{kDa}$ major outer dense fiber protein Odf84. Leucine zippers mediate associations of Odf84 and Odf 27. J. Biol. Chem. 272: 6105-6113.

Strom-Hansen, T., C. Cornatt and J. Jaroszewski, 1989. Interaction of gossypol with amino acids and peptides as a model of enzyme inhibition. Int. J. Peptide Res. 34: 306-310.

Swan, M.A., R. Vishwanath, I.G. White and P.D. Brown-Woodman, 1990. Electron microscopic observations on the effect of gossypol on rat cauda epididymis. Z. Mikrosk. Anat. Forsch. 104: 273-286.

Swierstra, E.E. and R.H. Foote, 1965. Duration of spermatogenesis and spermatozoon transport in the rabbit based on cytological changes, DNA synthesis and labeling with tritiated thymidine. Am. J. Anat. 116: 401412.

Taha, T.A., W.F. Shaaban, A.R. EL-Mahdy, F.D. EL-Nouty and M.H. Salem, 2006. Reproductive toxicological effects of gossypol on male rabbits: Semen characteristics and hormonal levels. Anim. Sci. 82: 259-269.

Taha, T.A., W.F. Shaaban, F.D. EL-Nouty and M.H. Salem, 2008. Molecular approach of gossypol - induced reproductive toxicity in male rabbits. Changes in seminal plasma amino acids and fatty acids. Animal 2: 1346-1352.

Teng, C.S., 1992. Biological activity in the repopulating rat spermatocyte after the withdrawal of gossypol treatment.III. The activity for the transcription of messenger RNA. Contraception 45: 167-176.

Teng, C.S., 1995. Biological activity in the repopulating rat spermatocyte after the withdrawal of gossypol treatment. V. Inhibition and recovery of microtubular dynein. Contraception 51: 313-318.

Teng, C.S., 1997. Reversible changes in the content of cellular and microtubular tubulin in spermatogenic cells after gossypol treatment. Contraception 55: 183-188.

Teng, C.S., N.Y. Yang and Y. Chen, 1995. Biological activity in the repopulating rat spermatocyte after the withdrawal of gossypol treatment. VI. Alteration in nuclear factors for interaction with histone gene promoter. Contraception 52: 129-134.

Velasquez-Pereira, J., P.J. Chenoweth, L.R. McDowell, C.A. Risco, C.A. Staples, D. Prichard, F.G. Martin, M.C. Calhoun, S.N. Williams and N.S. Wilkinson, 1998. Reroductive effects of feeding gossypol and vitamin E to bulls. J. Anim. Sci. 76: 2894-2904.

Vera, J.C., M. Brito and L.O. Burzio, 1987. Biosynthesis of rat sperm outer dense fibers during spermiogenesis. In vivo incorporation of $[3 \mathrm{H}]$ leucine into the fibrillar complex. Biol. Reprod. 36: 193202.

Wang, J.M., L. Tao, X.L. Wu, L.X. Lin, J. Wu, M. Wang and G.Y. Zhang, 1992. Differential binding of $(+)$ and (-) gossypol to plasma protein and their entry into rat testis. J. Reprod. Fert. 95: 277-282.

Williamson, R.A., J.K. Koehler, W.D. Smith and M.A. Stenchever, 1984. Ultrastructural sperm tail defects associated with sperm immotility. Fertil. Steril. 41: 103-107.

Zavos, P.M., J.R. Correa, C.S. Karagounis, A. Ahparaki, C. Phoroglou, C.L. Hicks and P.N. Zarmakoupis-Zavos, 1998. An electron microscope study of the axonemal ultrastructure in human spermatozoa from male smokers and nonsmokers. Fertil. Steril. 69, 3: 430-434. 
Table 1. Ultrastructural assessment of spermatozoa from control and gossypol-treated male rabbits

\begin{tabular}{|c|c|c|}
\hline Semen parameters & Control & Gossypol-treated \\
\hline Reacted acrosome $(\%)$ & $11.68 \pm 0.44^{\mathrm{b}}$ & $25.79 \pm 1.63^{\mathrm{a}}$ \\
\hline Connecting piece & Intact & Lesioned \\
\hline Segmented columns & Intact & Missing of some segmented colums \\
\hline Cytoplasmic droplets (\%) & $24.90 \pm 1.49^{b}$ & $50.74 \pm 2.31^{\mathrm{a}}$ \\
\hline \multicolumn{3}{|l|}{ Midpiece } \\
\hline Cytoplasmic membrane & Intact & Ruptured or disappeared \\
\hline Mitocondria & Intact & Vacuolated or disappeared \\
\hline \multicolumn{3}{|l|}{ Axonemal fiber } \\
\hline Coarse outer fiber & Intact & Missing of some coarse outer fiber \\
\hline Fiber doublets & Intact & Missing of some fiber doublets \\
\hline Central fibers & Intact & Intact \\
\hline Principal piece & Normal & Normal \\
\hline
\end{tabular}
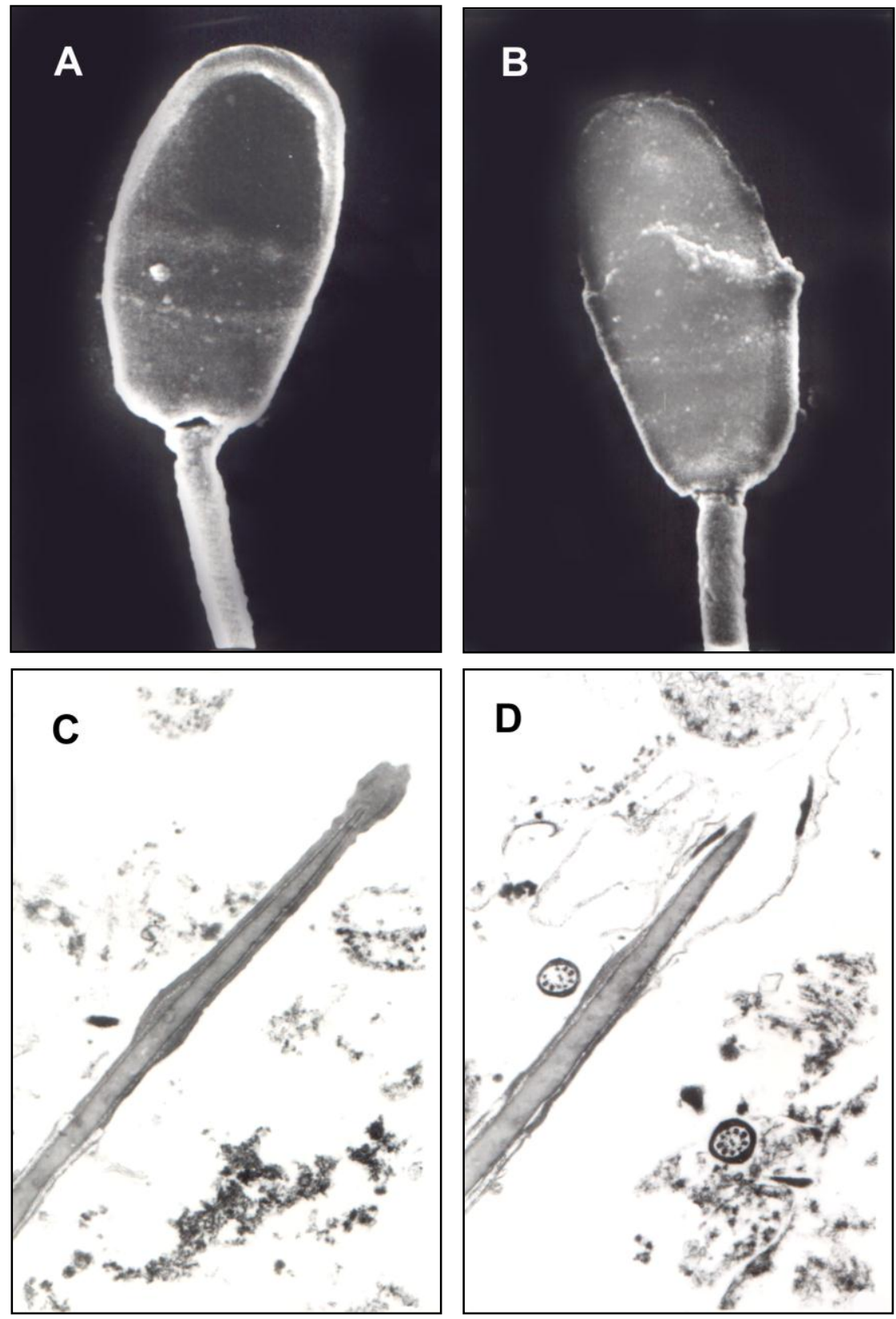

Fig. 1. Scanning and transmission electron micrographs of control and gossypol-treated rabbit sperm. A, C) control rabbit sperm showing intact acrosome. B, D). Gossypol-treated rabbit sperm revealing reacted acrosome. Original magnification $x 10000(A, B), x 13000$ (C) and $\times 15000$ (D). 

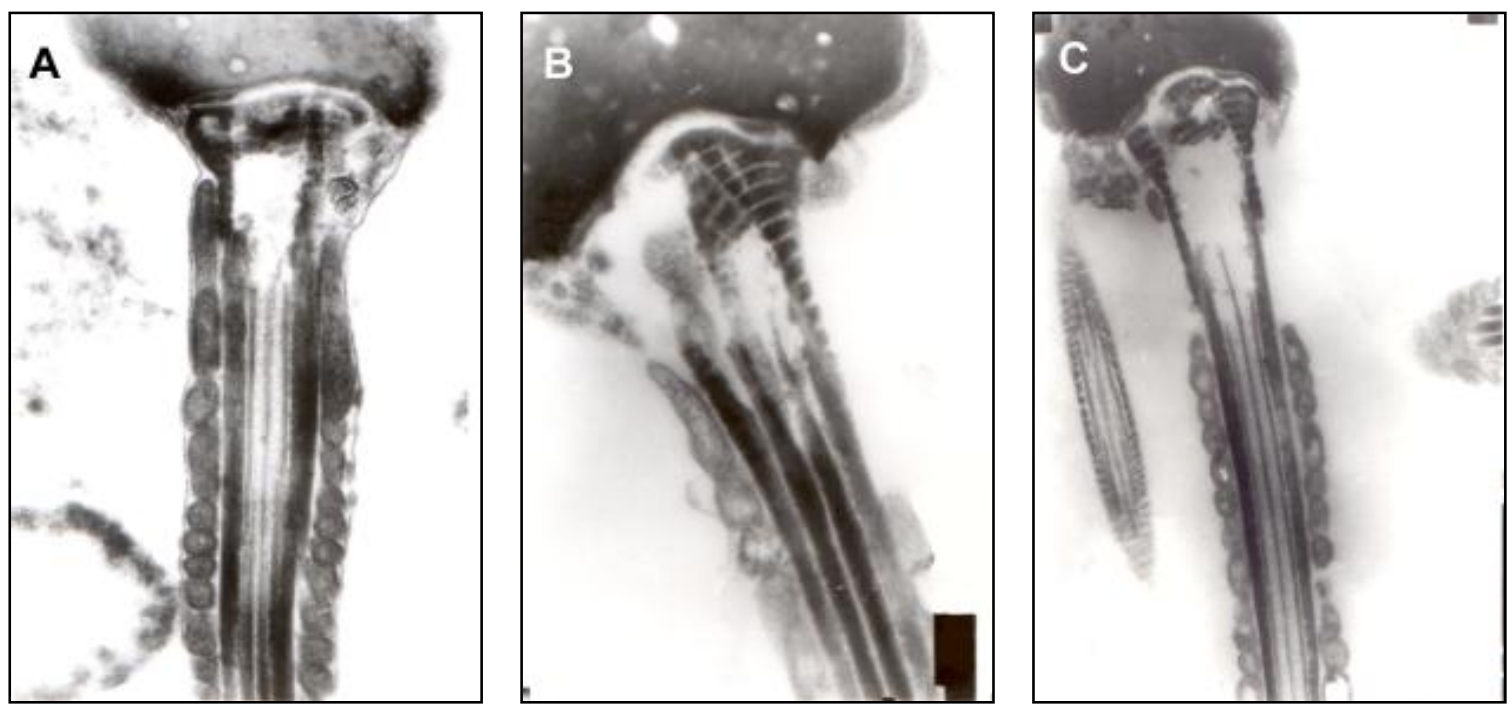

Fig. 2. Transmission electron micrographs of control and gossypol-treated rabbit sperm. A) Control rabbit sperm showing intact connecting piece and mitochondrial sheath. B, C) Gossypoltreated rabbit sperm illustrating lesion seen in the connecting piece expressed as degeneration of cytoplasmic membrane, and disappearance of some segmented columns (B) and complete removal of mitochondrial sheath near the neck region $(C)$. Original magnification $\times 25000(A)$, $x 40000(B)$ and $\times 20000(C)$.
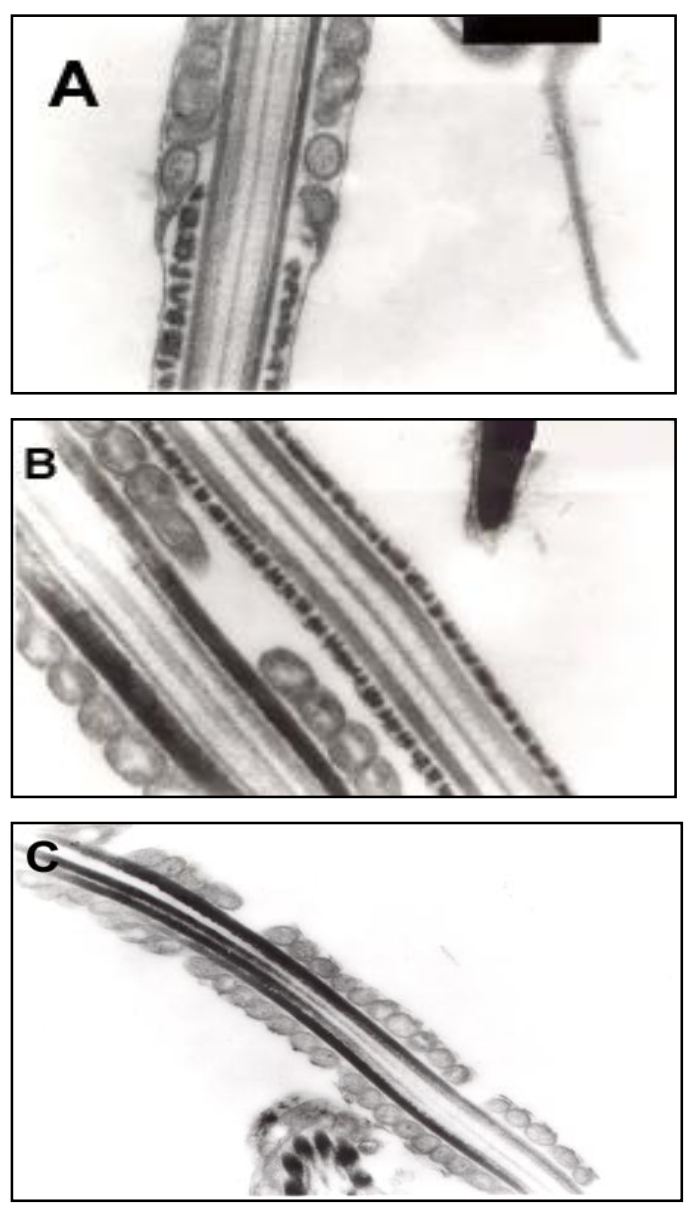

Fig. 3. Ultrastructure of longitudinal section of rabbit sperm through midpiece and principal piece in control rabbit sperm (A) and in gossypol-treated sperm (B, C) illustrating gossypolinduced disappearance of cytoplasmic membrane and degeneration of mitochondria $(C)$, but the principal piece seems normal $(B)$ and degeneration of cytoplasmic membrane and interruption in the continuation of the mitochondria (C). Original magnification $\times 40000(A, B)$ and $\times 20000(C)$. 

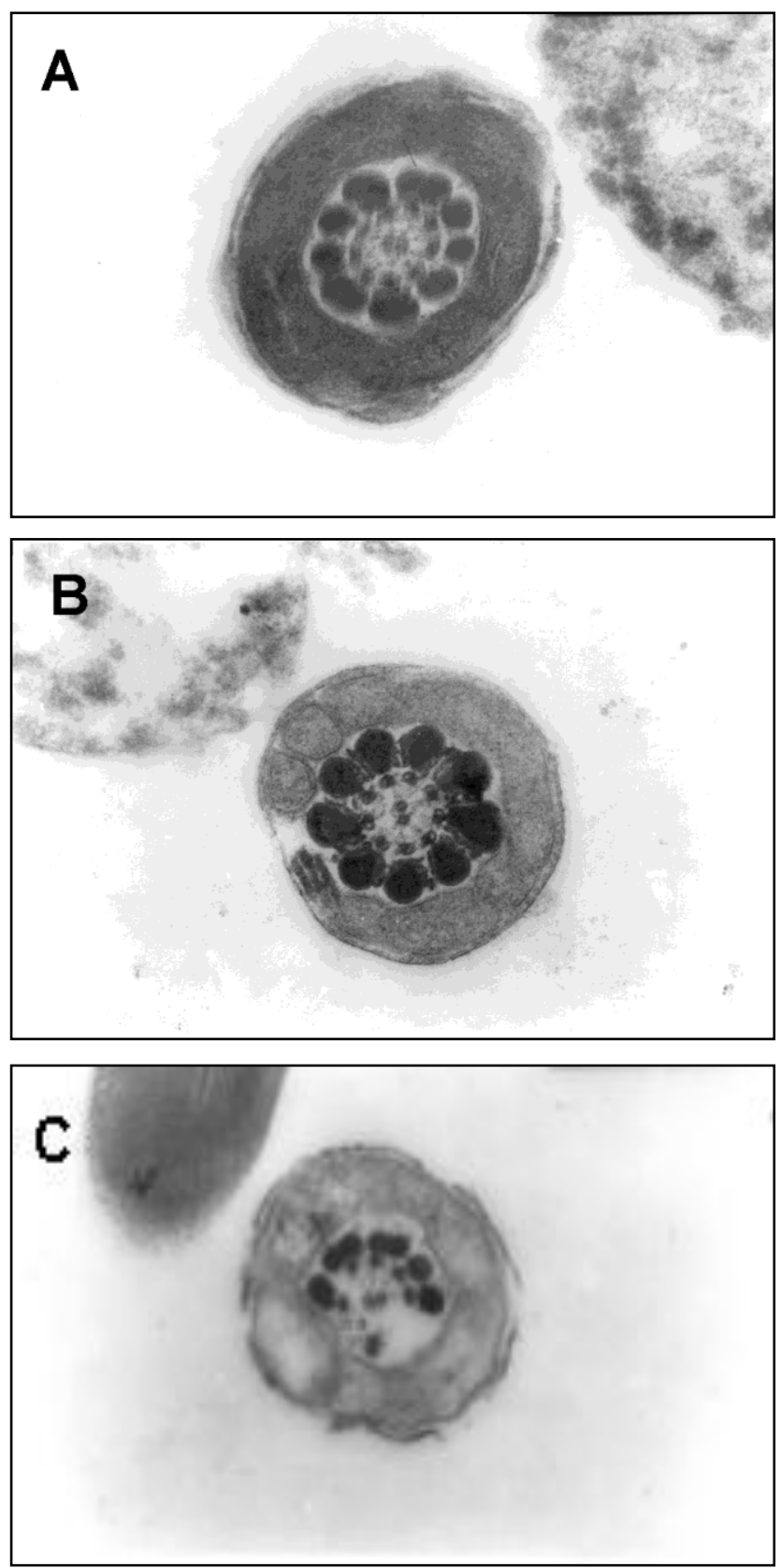

Fig. 4. Ultrastructural arrangement of cross-section in rabbit sperm midpiece in control rabbit sperm (A) revealing the normal appearance of mitochondrial sheath, outer dense fibers and axoneme; and in gossypol-treated rabbits $(\mathrm{B}, \mathrm{C})$ showing degeneration of mitochondria $(\mathrm{B})$ and rupture of cytoplasmic membrane, extensive vacuolation of mitochondria and disappearance of some outer dense fibers and fiber doublets (C). Original magnification x50000 (A, B, C). 

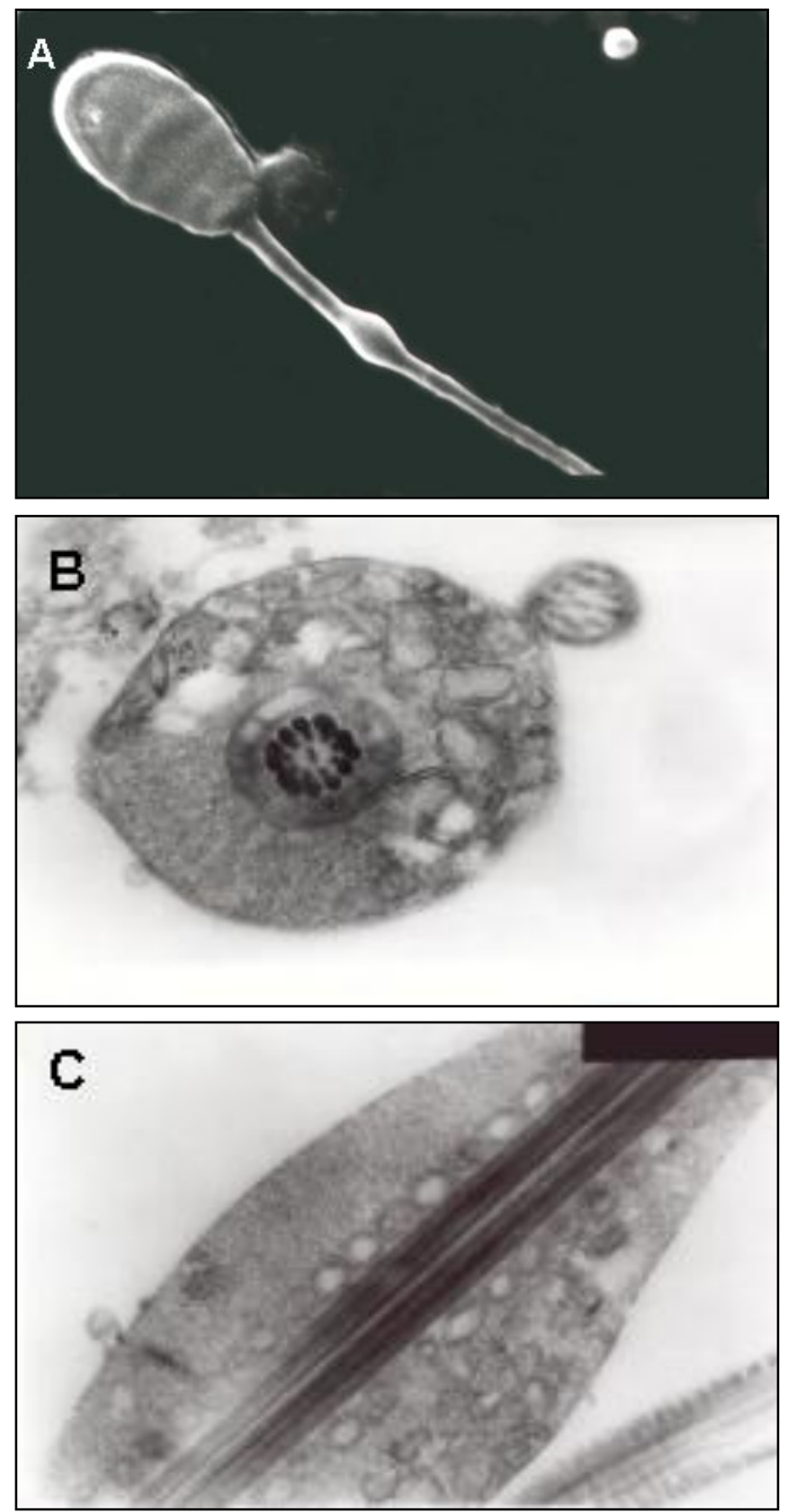

Fig. 5. Cytoplasmic droplets of gossypol-treated rabbit sperm. A) Overview of gossypol-treated rabbit sperm illustrating a cytoplasmic droplet. B, C) Transmission electron micrographs of cross (B) and longitudinal sections (C) of cytoplasmic droplet revealing extensive vacuolation and loss of cristae of mitochondria. Note the presence of numerous vesicles in the cytoplasmic droplet and its hollow appearance. Original magnification X5000 (A) and X30000 (B, C). 
التثوهات في التراكيب الاقيقة في الحيو انات المنوية في الأرانب المعاملة بالجوسيبول

ولاء فؤاد شعبان، طه أحمد طه، فرحات الدسوقي النوتي، محمد حلمي سالم

قسم الإنتاج الحيواني، كلية الزراعة (الشاطبي)، جامعة الإسكندرية، الإسكندرية، جمهورية مصر العربية

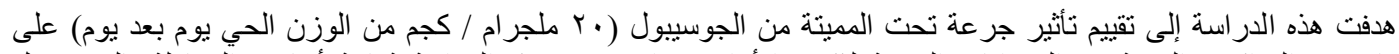

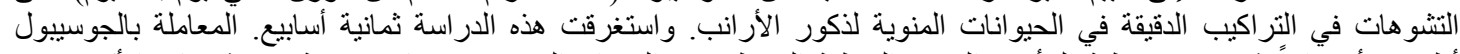

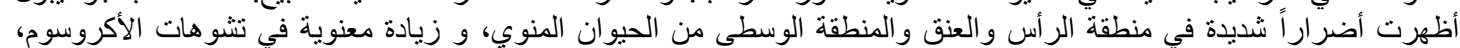

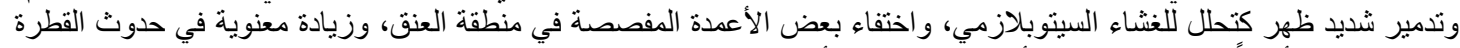

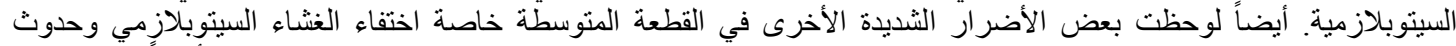

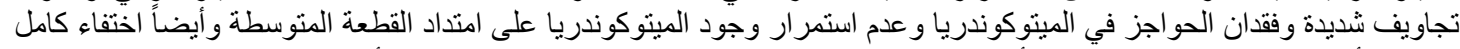

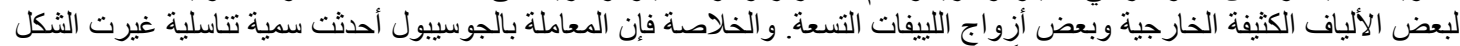

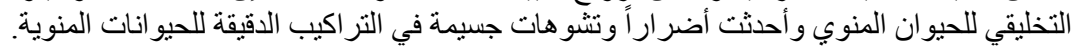

